\title{
Tracing dynamic nuclear polarization pathways using transition metal - nuclear spin rulers
}

\author{
Sheetal Kumar Jain ${ }^{a}$, Chung-Jui Yu ${ }^{b}$, C. Blake Wilsonc, Tarnuma Tabassumª, Danna E. Freedman ${ }^{b}$, Songi Han ${ }^{a, d}{ }^{*}$ \\ a. Department of Chemistry and Biochemistry, University of California, Santa Barbara, CA 93106-9510, USA. \\ b. Department of Chemistry, Northwestern University, Evanston, IL 60208, USA. \\ c. Department of Physics, University of California Santa Barbara, Santa Barbara, CA 93106-9510, USA. \\ d. Department of Chemical Engineering, University of California Santa Barbara, Santa Barbara, CA 93106-9510, USA.
}

Dynamic nuclear polarization (DNP) experiments were performed on a set of transition metal $\left(\mathrm{V}^{4+}\right)$-nuclear spin $\left({ }^{1} \mathrm{H}\right)$ rulers with isolated $\mathrm{V}^{4+}{ }^{1} \mathrm{H}$ distances tuned between 4 - $12.6 \AA$ using the endogenous $\mathrm{V}^{4+}$ ions as polarizing agents to experimentally determine the size of the spin diffusion barrier and its impact on DNP pathways. We report DNP enhancements of up to $\varepsilon_{\text {chirp-DNP }} \sim 33$ by utilizing a chirp microwave pulse train to enhance the saturation of the broad EPR spectrum of $\mathrm{V}^{4+}$ ions. The rate of ${ }^{1} \mathrm{H}$ polarization buildup was used to carefully decouple the roles of direct $\mathrm{V}^{4+}{ }^{1} \mathrm{H}$ polarization transfer and ${ }^{1} \mathrm{H}-{ }^{1} \mathrm{H}$ spin diffusion processes. Experiments reveal the spin diffusion barrier for the proton spins to be at a distance between 4.0 and $6.4 \AA$ from the $\mathrm{V}^{4+}$ center under DNP conditions, while ${ }^{1} \mathrm{H}$ spins as far away as $12.6 \AA \AA$ were directly polarized by DNP and detected by NMR. We observed a gradual change in the DNP buildup rate with distance between the paramagnetic center and the nearest ${ }^{1} \mathrm{H}$ spins ranging between 4.0 and $12.6 \AA$, demonstrating the rate-limiting effect of the spin diffusion barrier on ${ }^{1} \mathrm{H}$ nuclear spin diffusion up to at least 12.6 from the $\mathrm{V}^{4+}$ metal center. This study paves the way towards hyperfine DNP spectroscopy that achieves detection and diagnosis of select nuclear spins at a set distance from the transition metal ion. This study also represents the first use of $\mathrm{V}^{4+}$ transition metal as a polarizing agent, significantly expanding the scope of DNP. Arbitrary waveform generation and wide frequency tunability of a solid-state microwave source extends the capacity of DNP to wide line EPR transition metal centers endogenous to catalysts, energy materials and metalloenzymes.

\section{Introduction}

Dynamic nuclear polarization (DNP) is the most broadly applicable hyperpolarization method to enhance the nuclear magnetic resonance (NMR) signal, relying on polarization transfer from highly polarized electron spins $(e)$ to the surrounding nuclear spins ( $n$ ). In typical DNP experiments for biological or material studies, a source of unpaired electron spins known as a polarizing agent (PA) is added to the NMR sample, together with a cryo-protectant to generate a glassing matrix. Microwave $(\mu \mathrm{w})$ irradiation near the electron paramagnetic resonance (EPR) frequency of the polarizing agent can drive polarization transfer from the electron spins to the surrounding nuclear spins. DNP has already transformed the scope of NMR spectroscopy and imaging at the current state of the art. $^{2}$

Nitrogen-centered nitroxide or carbon-centered trityl radicals are used nearly exclusively as PAs, owing to their favorable properties including stability, solubility, molecular geometry, relatively long electron spin relaxation time and near 2.0 electron spin $g$ factor, matching that of a free electron. ${ }^{2}$ The use of paramagnetic transition metal centers endogenous to the molecule or material of interest would dramatically broaden the scope of DNP to boost NMR sensitivity and yield critical structural information via selective enhancements of proximal nuclear spins. To date, only high spin paramagnetic metals such as $\mathrm{Gd}^{3+}$, $\mathrm{Mn}^{2+}$, and $\mathrm{Cr}^{3+}$ with narrow central EPR transition band have been used as PAs for high-field (>5 T) DNP ${ }^{3-6}$ and few select studies relied on endogenous paramagnetic metal centers of materials or metalloenzymes. ${ }^{7-8}$ Transition metals ions, such as $\mathrm{Ni}^{+}, \mathrm{Cu}^{2+}, \mathrm{Ni}^{3+}$ and $\mathrm{V}^{4+}$ that are widely present and central to the function of battery materials, catalytic compounds and biological enzyme complexes are considered inaccessible for DNP due to their wide EPR lines and g-values significantly shifted from 2 . 
However, to utilize endogenous paramagnetic centers for DNP, it is critical to understand their detailed impact on the NMR signal of proximal nuclear spins. The paramagnetic effects such as paramagnetic relaxation enhancement (PRE) $)^{9-11}$, contact and pseudo-contact shifts (CS and PCS) ${ }^{12-14}$, have significant influence on the NMR spectra. The PRE effects have been used to extract critical structural information in biomolecules. ${ }^{15}$ However, under DNP conditions these effects cause nuclear spins located too close to the paramagnetic centers to relax faster, such that their NMR spectra are broadened out. This leads to "paramagnetic quenching" that creates a forbidden zone, difficult to detect with NMR, near the paramagnetic centers. NMR signal quenching due to the paramagnetic centers has been observed under DNP conditions on various commonly used radicals at $5 \mathrm{~T}$ field and $84 \mathrm{~K}$ temperature as shown by Corzilius et al. ${ }^{16}$ McDermott and coworkers studied the paramagnetic effects of specifically bound biradicals on protein samples using high affinity biradical tags ${ }^{17-}$ 18 These effects have been recently used to obtain high-resolution DNP enhanced spectra using selective DNP. ${ }^{19}$ The PCS and CS effects cause a drastic change in the NMR chemical shift for the adjacent nuclear spins as a function of their distance to the paramagnetic metal center. This shift in frequency hinders the nuclear spin diffusion process from these nuclear spins to spins located outwards, thus forming a "spin diffusion barrier" somewhere around the paramagnetic center. ${ }^{20}$ The exact size of the spin diffusion barrier is a critical parameter in determining the polarization pathway and the DNP buildup rate because it determines the location of the closest nuclear spins that serves as a conduit for nuclear spin diffusion to remote nuclei. This parameter has been used in a number of studies that rely on numerical simulations and rate equations to understand the process of bulk nuclear polarization buildup in DNP and predict the buildup rate..$^{21-24}$ In a recent study, Tan et a ${ }^{25}$ determined such a spin diffusion barrier for ${ }^{1} \mathrm{H}$ near Trityl radicals in aqueous solvent to lie below $6 \AA$. However, the rate of nuclear spin diffusion is affected by the proximity of the paramagnetic center to nuclear spins located beyond the spin-diffusion barrier, creating a gradient of nuclear spin diffusion rates. These rates play critical roles in determining the buildup rate and hence sensitivity enhancement in DNP.

In this study, we first demonstrated $\mathrm{V}^{4+}$ ions as PAs to enhance ${ }^{1} \mathrm{H}$ NMR signal at $6.9 \mathrm{~T}$, where the EPR lines of the metal center spanned more than $3 \mathrm{GHz}$. A home-built DNP NMR instrument ${ }^{26-27}$ capable of frequency tuning across a broad range spanning $~ 10$ $\mathrm{GHz}$, arbitrary waveform generated (AWG) pulse shaping of the $\mu \mathrm{w}$ for broad-band saturation of the $\mathrm{V}^{4+}$ EPR line, and liquid helium operation to slow the electron spin relaxation of the $\mathrm{V}^{4+}$ ions made this study possible. Broadband saturation of the EPR line with chirped microwave irradiation significantly enhanced the DNP performance at $4 \mathrm{~K}$. Note that the $g$ anisotropy of transition metal centers can be extremely large, larger than that of $\mathrm{V}^{4+}$, spanning more than tens of $\mathrm{GHz}$. As such, this study is a first and critical step towards establishing access to wide-line transition metal centers by DNP. We designed a series of vanadyl complexes with deliberately installed ${ }^{1} \mathrm{H}$ containing propyl groups at varying distances from the $\mathrm{V}^{4+}$ center on an otherwise nuclear spin-free ligand backbone. ${ }^{1}$ These transition metal-nuclear spin rulers offered us the opportunity to systematically explore ${ }^{1} \mathrm{H}$ spin diffusion mechanisms as a function of distance relative to the spin-diffusion barrier. The size of the spin-diffusion barrier was determined to be between $4.0 \AA$ and $6.4 \AA$, consistent with previously hypothesized values. ${ }^{1,}{ }^{25}$ The effect of the $V^{4+}{ }^{1} H$ coupling strength and nuclear spin-diffusion rate on the DNP process were elucidated with electron spin relaxation and DNP buildup rate measurements. Direct DNP of ${ }^{1} \mathrm{H}$ spins at $12.6 \AA$ away from the $\mathrm{V}^{4+}$ center was achieved with a characteristic buildup time determined by the $\mathrm{V}^{4+}{ }^{1} \mathrm{H}$ distance and the nuclear spin diffusion process. The results of our DNP work, based on the hyperfine interaction between the metal center and the proximal nuclear spins, provide the initial step towards critical structural and dynamical information around paramagnetic active sites and function centers. We coin this novel category of experiments hyperfine DNP spectroscopy. In contrast to hyperfine EPR, hyperfine DNP directly detects the 
NMR signal and so resolves NMR chemical shifts and line-shapes. Additionally, unlike paramagnetic NMR, this technique benefits from signal enhancements originating from the transition metal center.

\section{Materials and methods}

The solution of the four vanadyl complexes (see Figure 1a) in $99.5 \%$ deuterated dimethylformamide (DMF) solvent were prepared as described in a previous publication by Graham et al. ${ }^{1}$ The $\mathrm{V}^{4+}$ ion concentrations for the complexes in saturated solutions are $10.5 \mathrm{mM}$ for $\mathbf{1}, 77.2 \mathrm{mM}$ for $2,14 \mathrm{mM}$ for 3 and $13.2 \mathrm{mM}$ for 4 (see section 6 of the ESI). The saturated solutions were flame sealed in EPR quartz tubes of length $\sim 2 \mathrm{~cm}$, with inner and outer diameters of $2.2 \mathrm{~mm}$ and $3.0 \mathrm{~mm}$, respectively, to avoid any air exposure. In case of complex 2, a sample with $13 \mathrm{mM}$ $\mathrm{V}^{4+}$ concentration was also prepared to confirm the observed trends in buildup rate at comparable concentration.

The DNP NMR experiments were performed with a broad-band quasi-optics (QO) based dual DNP-EPR instrument, operating at $6.9 \mathrm{~T}$ magnet and at 4-10 K. Details of the home-built DNP instrumentation have been described previously. ${ }^{26-28}$ All static ${ }^{1} \mathrm{H}$ NMR experiments were recorded using the solid echo (90x$\mathrm{t}-90 \mathrm{y})$ pulse sequence shown in Figure 2a. To perform DNP experiments, the samples were freeze-quenched in liquid nitrogen for glass formation, and then cooled to $4 \mathrm{~K}$ for the experiments.

\section{Results and discussion}

The $\mathrm{V}^{4+}{ }^{1} \mathrm{H}$ rulers, i.e. the vanadyl complexes with controlled average $\mathrm{V}^{4+}{ }_{-}{ }^{1} \mathrm{H}$ distances $\left(R_{\mathrm{V}-\mathrm{H}}\right)$, are shown in Figure 1a, with $R_{\mathrm{V}-\mathrm{H}}=4.0 \AA$, $6.6 \AA$, $9.3 \AA$ and $12.6 \AA$ for complexes 1-4, respectively. Tetraphenylphosphonium cation was used as counter ion to balance the charge, and the solvent was deuterated dimethylformamide (DMF). ${ }^{1} \mathrm{H} \quad \mathrm{NMR}$ spectra were acquired of complex $\mathbf{4}$ with protonated (4) and deuterated (4') tetraphenylphosphonium cation.

The field-swept echo detected EPR spectra of the vanadyl complexes were recorded at a $\mu \mathrm{w}$ frequency $\left(\omega_{\mu w}\right)$ of $240 \mathrm{GHz}$ by sweeping the field from $8.4 \mathrm{~T}$ to
$9 \mathrm{~T}$ at $5 \mathrm{~K}$ (see Figure $\mathrm{S} 1$ in the ESI). The principle components of $g$ and hyperfine coupling (A) of the ${ }^{51} \mathrm{~V}$ paramagnetic center were extracted by fitting the spectra using EasySpin. ${ }^{29}$ Figure $1 \mathrm{~b}$ depicts the EPR line-shapes simulated at $6.9 \mathrm{~T}$ based on these experimental parameters. The $g$ and A tensor values found for all vanadyl complexes are well resolved at the employed high field and frequency, and in agreement with the previously reported values for the same complexes determined by X-band CW EPR analysis (Table S1, S2 in ESI). ${ }^{1}$ The EPR lines of the vanadyl complexes span more than $3 \mathrm{GHz}$, which are significantly broader compared to the nitroxide-based radicals that span $0.6-1 \mathrm{GHz}$ at $6.9 \mathrm{~T}$ and $4 \mathrm{~K}$.
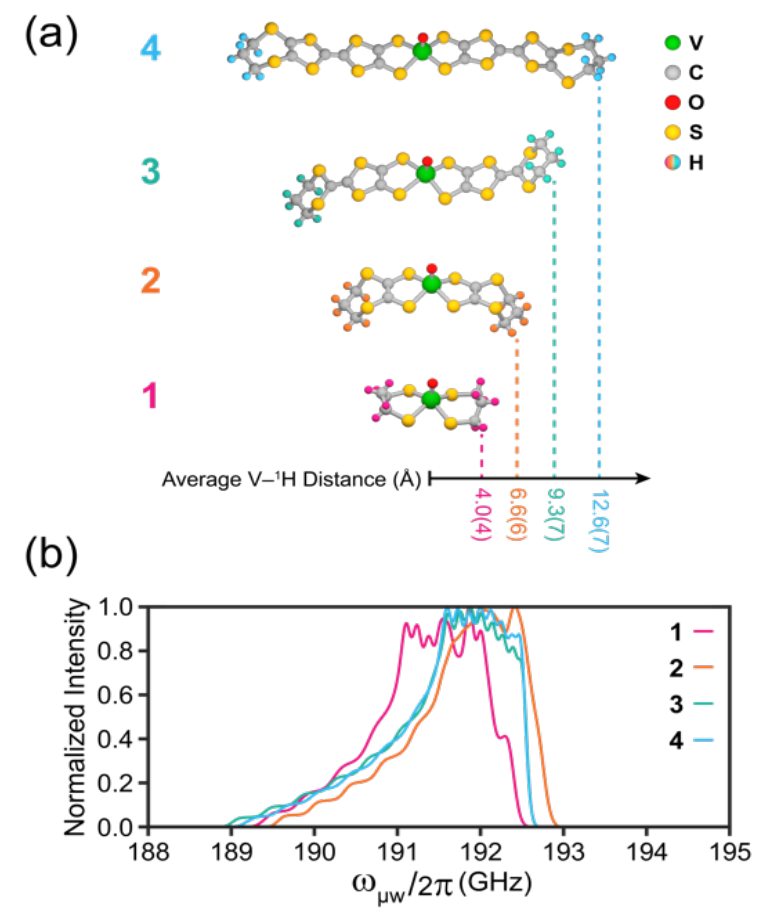

Figure 1 (a) Structures of the four $\mathrm{V}^{4+}+1 \mathrm{H}$ rulers presented in Graham et $\mathrm{al}^{1}$ that were used in this study. (b) The EPR lineshapes of the complexes 1-4, simulated for $6.9 \mathrm{~T}$ field using the experimental data from $240 \mathrm{GHz}$ CW EPR spectra at $5 \mathrm{~K}$ in magenta, orange, green and cyan respectively.

To systematically study the effect of $R_{\mathrm{V}-\mathrm{H}}$ on the DNP performance and the spectral feature of ${ }^{1} \mathrm{H} N M R$, DNP experiments were performed on the four vanadyl complexes with the endogenous $V^{4+}$ centers as the polarizing source. The DNP frequency profiles of these broad line $\mathrm{V}^{4+}$ centers were recorded by varying the $\mu \mathrm{w}$ irradiation frequency over a $3 \mathrm{GHz}$ span, relying on 
the broad-band QO-DNP instrument and using the NMR pulse sequence shown in Figure 2a. The NMR signal enhancement factors were determined by calculating the ratio $\varepsilon=\left(S_{O N}-S_{O F F}\right) / S_{\text {OFF }}$, where $S_{\text {ON }}$ and $S_{\text {OFF }}$ are NMR signal intensities under $\mu \mathrm{w}$ on and $\mu \mathrm{w}$ off conditions at equal buildup times.

(a)

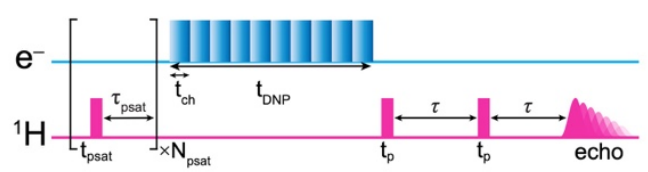

(b)

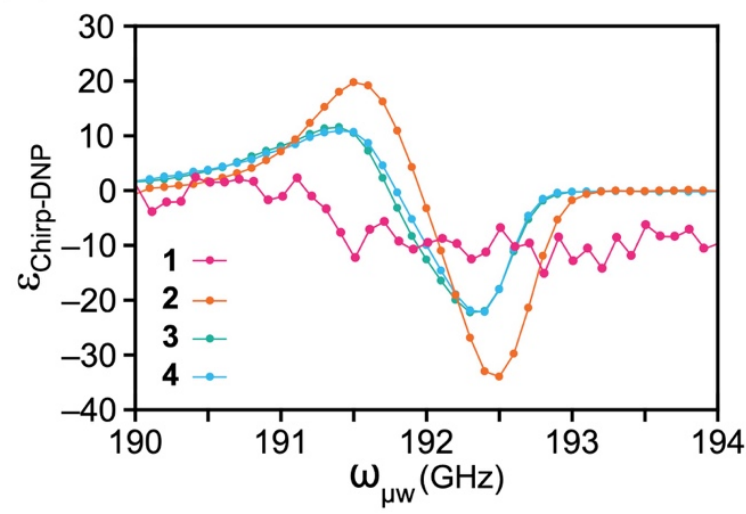

Figure 2 (a) Solid-echo pulse sequence used to collect ${ }^{1} \mathrm{H} N M R$ signals, with the microwaves on for DNP and off for normal NMR experiments. (b) DNP frequency profiles for 1-4 in magenta, orange, green and cyan respectively. The experiments were performed at $4 \mathrm{~K}$ on a $6.9 \mathrm{~T}$ magnet with using chirped $\mu \mathrm{w}$ pulses with the parameter $\Delta \omega_{\mathrm{ch}}=200 \mathrm{MHz}, \mathrm{t}_{\mathrm{ch}}=300 \mu \mathrm{s}$, $\mathrm{t}_{\text {buildup }}=60 \mathrm{~s}$, $\mathrm{P}_{\mu \mathrm{w}}=120 \mathrm{~mW}$, and the interpulse delay $\tau=50 \mu \mathrm{s}$.

Figure $2 \mathrm{~b}$ shows the DNP frequency profiles recorded with chirped $\mu \mathrm{w}$ pulse trains with a buildup time $t_{D N P}=60 \mathrm{~s}$ and $\mu \mathrm{w}$ power $P_{\mu w}=120 \mathrm{~mW}$. The chirped $\mu \mathrm{w}$ pulse train yielded maximum $\varepsilon_{\text {Chirp-DNP }}$ with a chirp pulse length of $\left(t_{c h}\right)=300 \mu$ s and sweep width $\left(\Delta \omega_{c h}\right)=300 \mathrm{MHz}$ that was repeated continuously throughout $t_{D N P}$. The experimental DNP frequency profiles were found to be broad and asymmetric, the maximum positive and negative enhancement positions $\sim 1 \mathrm{GHz}$ apart and the negative enhancements at around $192.5 \mathrm{GHz}$ found to be greater than the positive enhancements at around 191.5 GHz for all cases. The same general features of the DNP profiles were observed with monochromatic CW DNP, except with lower enhancement values ( $\varepsilon_{\mathrm{cW}}$ ${ }_{D N P}$ ), as shown in Figure S2 of the ESI. The features of the DNP profiles are directly reflected in the EPR patterns that display a sharp increase in intensity at the high frequency end and a longer tail towards low frequencies for all complexes due to broad dispersion of the EPR line caused by inhomogeneous broadening (see Figure 1b). The features of the DNP frequency profiles indicate that the underlying mechanism is differential solid-effect $(\mathrm{SE}),{ }^{30}$ in which the positive and negative enhancements overlap due to an inhomogeneously broadened EPR line. Hence, the overall shape of the DNP profile deviate from that of SE obtained with a narrow EPR line, while the separation between the maximum enhancement positions is significantly larger than two times the nuclear Larmor frequency, given the wide distribution of electron spin frequency packets across the inhomogeneously broadened EPR line.

It is important to note that the enhanced ${ }^{1} \mathrm{H}$ NMR signal observed can come from three different sources: the vanadyl complexes, the counter ions and the $0.5 \%$ protons in the DMF solvent. In order to trace the polarization pathways in DNP, we conducted a careful analysis of the ${ }^{1} \mathrm{H}$ NMR spectra and DNP buildup time. A comparison of the ${ }^{1} \mathrm{H}$ NMR spectra recorded with chirped $\mu \mathrm{w}$ pulses (solid-line), $\mathrm{CW} \mu \mathrm{w}$ irradiation (dashed-line) and the $\mu \mathrm{w}$ off signals (dotted-line) is shown in Figure 3 in magenta, orange, green and cyan colors, respectively, for 1-4. The experimental conditions and parameters are the same as those for the DNP frequency profiles shown in Figure $2 b$, with $\omega_{\mu w}$ set to the frequency of the positive maximum enhancement in all cases. The values for $\varepsilon_{\mathrm{CW} \text {-DNP }}$ were found to be $\sim 0.5,1.7,1.7$ and 1.8 , and for $\varepsilon_{\text {Chirp-DNP }} \sim 1,19,12$ and 9 for $1-4$ respectively at the frequency generating positive maximum DNP enhancement. The corresponding spectra for the negative maximum enhancement recorded by chirpDNP are shown in the ESI Figure S3. In all cases except for 1 , the DNP performance increases significantly from CW monochromatic to broad-band chirp train $\mu \mathrm{w}$ irradiation. The superiority of the chirped $\mu \mathrm{w}$ pulse trains over CW irradiation has been reported recently by Kaminker et al. ${ }^{31} \mathrm{~A}$ gain factor $\left(\varepsilon_{\mathrm{Chirp}-\mathrm{DNP}} / \varepsilon_{\mathrm{CW}-\mathrm{DNP}}\right.$ ) between 4 to 5 was reported with various nitroxide based radicals at temperatures $4 \mathrm{~K}$ and $25 \mathrm{~K}$ owing to 
more efficient EPR saturation achieved by frequency sweeping with a chirp pulse train. Broad-band pulse trains are expected to dramatically benefit DNP of transition metals, which is evident in this study as the EPR spectra of $\mathrm{V}^{4+}$ span several $\mathrm{GHz}$. The gain factor was $\varepsilon_{\text {Chirp-DNP }} / \varepsilon_{C W-D N P}>4$ in all cases for $1-4$, and reaching up to $\sim 10$ for 2 ( $\varepsilon_{\text {Chirp-DNP }}=-33$ and $\varepsilon_{C W-D N P}=-$ 3.5 in the region of negative enhancement). The higher enhancement observed in sample $\mathbf{2}$ is attributed to the high solubility of the complex in the DMF solvent $(77.2 \mathrm{mM}),{ }^{32}$ resulting in a relatively large number of $\mathrm{V}^{4+}$ metal center in the sample. The difference in signal to noise ratios (SNRs) of the $\mu \mathrm{w}$ off ${ }^{1} \mathrm{H}$ NMR signals among the complexes is a result of the varying solubility of the compounds.

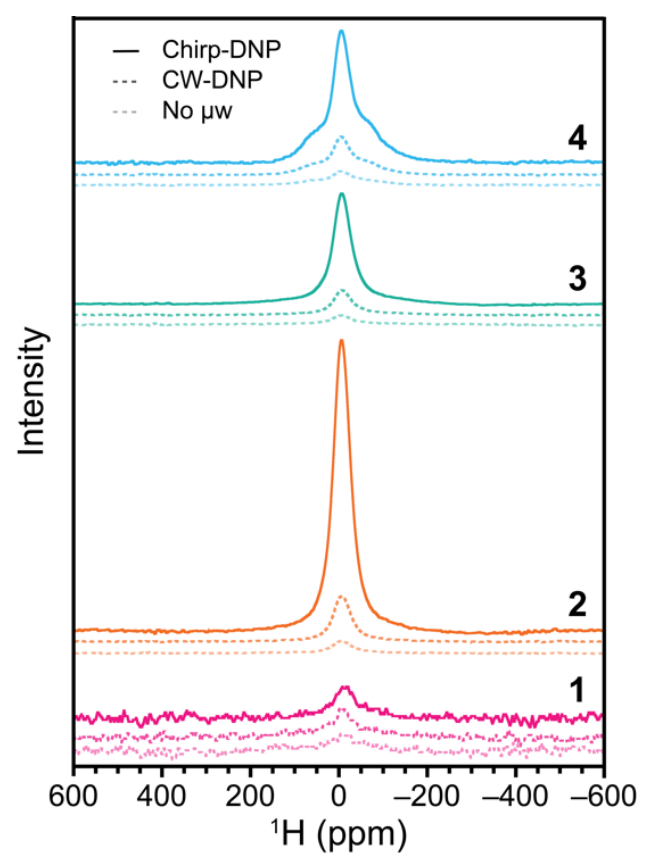

Figure $3{ }^{1} \mathrm{H}$ NMR spectra of the four complexes acquired under $\mu \mathrm{w}$ off (dotted-lines), and with CW (dashed-lines) and chirp DNP (solid-lines) are shown in magenta, orange, green and cyan colors for $\mathbf{1 - 4}$, respectively. $\omega_{\mu w}$ was set to positive maximum signal enhancement for each case while the rest parameters were same as Figure 2.

The spectral line-shapes of $\mathbf{4}$ clearly indicate the presence of two signals with different line widths, while there is only one narrow component present in the spectra of 1-3. In order to distinguish between the two signals in 4, the inter-pulse delay $(\tau)$ was varied between 30-200 $\mu$ s (see ESI Figure S4), such that the broad signal was suppressed and only the narrow signal survived. (a)

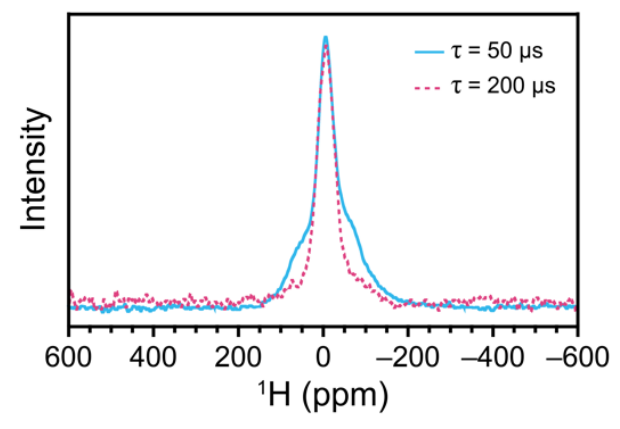

(b)

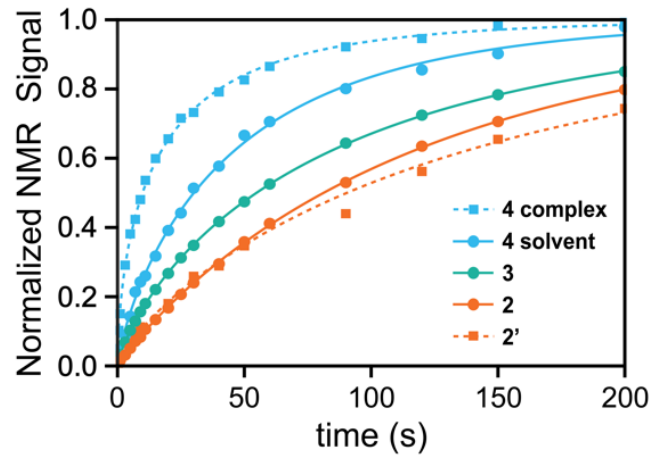

Figure 4(a) Chirp DNP enhanced ${ }^{1} \mathrm{H}$ NMR spectra from complex 4 with inter-pulse delays of $50 \mu \mathrm{s}$ (cyan, solid-line) and $200 \mu \mathrm{s}$ (magenta, dashed-line), recorded on a $6.9 \mathrm{~T}$ magnet at $4 \mathrm{~K}$ temperature. The spectrum with $\tau=200 \mu$ s was scaled up to match the peak heights of the two spectra to highlight the suppression of the broad component. (b) The bulk polarization buildup using chirp DNP experiments (plot markers) and fitted curves (lines) for the $\mathbf{2}$ at $77 \mathrm{mM}$ (orange-circles, 2) and 13mM (orange-square, 2'), $\mathbf{3}$ at 14 $\mathrm{mM}$ (green), and $\mathbf{4}$ at $13.2 \mathrm{mM}$ from the solvent protons (cyancircles) and complex protons (cyan-square). The fitted parameters are given in Table 1

Figure 4a shows the ${ }^{1} \mathrm{H}$ spectra of 4 recorded with $\tau=50 \mu$ s (cyan, solid-line) and $\tau=200 \mu$ s (magenta, dashed-line). The fast-relaxing broad component of the signal is assigned to the protons covalently attached to the complex, referred to as "complex protons", and the narrow signal with relatively long transverse relaxation time $\left(T_{2 n}\right)$ to the "solvent protons" that include the protons on the counter ions and the DMF solvent. As a control, we measured the solution-state ${ }^{1} \mathrm{H}$ NMR spectrum of $\mathbf{3}$ and $\mathbf{4}$, wherein we observed a broadening of the complex ${ }^{1} \mathrm{H}$ (see ESI Figure S5). The signal for complex protons at $2.22 \mathrm{ppm}$ are significantly broader compared to the counterion protons at $7.77 \mathrm{ppm}$ and the DMF protons at 7.91, 2.80 , and $2.64 \mathrm{ppm}$. This indicates that the complex ${ }^{1} \mathrm{H}$ are more affected by the paramagnetic effects than the solvent ${ }^{1} \mathrm{H}$. Fits to the ${ }^{1} \mathrm{H}$ NMR spectrum of 4 at $4 \mathrm{~K}$ 
acquired with short $\tau$ revealed a narrow peak at -4.3 ppm and an upfield shifted broad peak at $-15.3 \mathrm{ppm}$ (ESI Figure S6). The change in the peak positions are ascribed to paramagnetic effects and temperature, which has been previously observed in $S=1 / 2$ vanadium complexes..$^{33}$ The assignment of the broad peak to the complex ${ }^{1} \mathrm{H}$ and the narrow one to the solvent ${ }^{1} \mathrm{H}$ was further validated by comparing the low temperature NMR spectra of $\mathbf{4}$ sample with deuterated and protonated counter ions (ESI Figure S7). Though this data was acquired under different experimental conditions than the DNP data in Figure $4 a$, since the cryostat and probe was rebuilt, the key observation was the suppression of the narrow spectral component when deuterated counter ions were present. We attribute the observation of only the narrow component in samples 1-3 to the short $T_{2 n}$ values of the complex protons caused by the stronger paramagnetic relaxation enhancement (PRE) effect of the proximal $\mathrm{V}^{4+}$ center in these complexes with shorter $R_{V-H}$ compared to 4 . Experiments with a very short $\tau$ could be designed to detect those signals, but this would lead to artifacts caused by competing instrumentation dead time.

Next, we recorded the DNP buildup curves of the ${ }^{1} \mathrm{H}$ NMR signal of complexes $\mathbf{2 - 4}$ as shown in Figure $4 \mathrm{~b}$ for $\mathbf{2}$ in orange, $\mathbf{3}$ in green, and $\mathbf{4}$ in cyan. The buildup curves of the two components of $\mathbf{4}$ are plotted separately: cyan circles and solid-line correspond to the solvent protons, and cyan squares and dashed-line represent the complex protons. The complex proton buildup curve was obtained by taking the difference of spectra recorded using $\tau=50 \mu$ s and $\tau=200 \mu$ s for each data point. For $\mathbf{2}$, the buildup curves at complex concentration of $77.2 \mathrm{mM}$ (at saturation) and $13 \mathrm{mM}$ (comparable with the other three complexes) are shown in orange circles and orange squares, respectively. Note that 1 shows no DNP enhancement (Figure 3); hence the buildup curve could not be obtained due to the small SNR of the NMR signal.

All polarization buildup curves were fitted to a stretched exponential $I=I_{0}\left[1-e^{-\left(\frac{t}{T_{\text {DNP }}}\right)^{n}}\right]$, where $I_{0}, \mathrm{~T}_{\mathrm{DNP}}$ and $n$ were the fitting parameters. The value of the parameter $n(\leq 1)$ provides us with information about the nature of the buildup curve, i.e., whether it is a mono-exponential $(n \sim 1)$ process dominated by nuclear spin diffusion or a multi-exponential $(n<1)$ process in which the buildup rates are different based on the $\mathrm{V}^{4+}{ }_{-}{ }^{1} \mathrm{H}$ hyperfine coupling strengths. ${ }^{34} \mathrm{~T}_{\mathrm{DNP}}$ is the time constant for the polarization buildup and $I_{0}$ is the NMR signal intensity at DNP saturation. The fitted values for $T_{\mathrm{DNP}}, n$ and $I_{0}$ are given in Table 1 for the buildup curves.

Table 1 Fitted parameters for the proton build up curves in 2-4 for chirped DNP experiments.

\begin{tabular}{|c|c|c|c|}
\hline Complex\# & $\mathrm{T}_{\text {DNP }} / \mathrm{s}$ & $\mathrm{n}$ & $\mathrm{I}_{0}$ \\
\hline 2 & $120.4 \pm 9.0$ & $0.93 \pm 0.02$ & $1.253 \pm 0.048$ \\
\hline $2^{\prime}$ & $142.4 \pm 28$ & $0.80 \pm 0.09$ & $1.053 \pm 0.072$ \\
\hline 3 & $87.4 \pm 3.9$ & $0.78 \pm 0.01$ & $1.176 \pm 0.018$ \\
\hline 4 (solvent) & $47.7 \pm 6.5$ & $0.79 \pm 0.06$ & $1.021 \pm 0.049$ \\
\hline 4 (complex) & $18.2 \pm 1.5$ & $0.60 \pm 0.03$ & $1.003 \pm 0.020$ \\
\hline
\end{tabular}

The complex protons from 4 show the shortest buildup time ( $T_{\mathrm{DNP}}=18.2 \mathrm{~s}$ ) as these are strongly coupled to the $\mathrm{V}^{4+}$ centers; hence a direct $\mathrm{V}^{4+}{ }^{1} \mathrm{H}$ transfer governs this buildup time. This is also manifested in the stretching parameter $n=0.6$, which is smallest among the fitted $\mathrm{n}$ values and closest to 0.5 , indicating a multi-exponential buildup owing to contributions from multiple direct $\mathrm{V}^{4+}{ }^{1} \mathrm{H}$ couplings. In the polarization buildup process of the solvent protons of complex 4 , both direct $\mathrm{V}^{4+}{ }^{1} \mathrm{H}$ transfer and nuclear spin diffusion processes are involved; hence $n$ increases to 0.79 , indicating a more homogenous buildup process strongly influenced by nuclear spin diffusion. The polarization buildup times of the solvent protons increase systematically from $\mathbf{4}$ to $\mathbf{3}$ to $\mathbf{2}$, and eventually result in almost no DNP enhancement in $\mathbf{1}$, as the complex protons move closer to the paramagnetic metal center. Note that the $\mathrm{V}^{4+}$ concentration is highest in $\mathbf{2}$ due to its higher solubility. As a control, we compared the DNP buildup curves of complex 2 at $77 \mathrm{mM}$ and 2' at $13 \mathrm{mM}$ concentration, shown in Figure $4 \mathrm{~b}$ by the orange spheres and squares, respectively. Reassuringly, the results demonstrate that the observed spin dynamics and the buildup rates are not dictated by the concentration of the vanadyl complexes in this range. This also indicates that the DNP mechanism is still 
differential $\mathrm{SE}$, and that the spectral diffusion is negligible even at $77 \mathrm{mM}$ concentration, which we ascribe to the large $g$-anisotropy that makes spectral diffusion inefficient despite the high $\mathrm{V}^{4+}$ concentration. The general trend in $T_{\mathrm{DNP}}$ and $n$ from complex $\mathbf{2}$ to $\mathbf{4}$ was recapitulated by CW DNP as well (see Figure S8a and Table S3 in ESI). The $\mathrm{T}_{1 \mathrm{n}}$ relaxation times acquired without any $\mu \mathrm{w}$ irradiation also decrease from 2 to 4 (see Figure S8b in ESI for the buildup and fitting curves).

The observation that the $T_{D N P}$ values for the solvent protons are shortened from complex 2 (120.4 s) to 4 $(47.7 \mathrm{~s})$, even though the $R_{\mathrm{V}-\mathrm{H}}$ is increasing, shows that the process of nuclear polarization buildup by DNP here is not dictated by the direct $\mathrm{V}^{4+}{ }^{1} \mathrm{H}$ transfer process. Rather, this trend in the DNP buildup can be ascribed to the modulation in efficiency of the nuclear spin diffusion process by changes in the closest $\mathrm{V}^{4+}{ }^{1} \mathrm{H}$ distance. The complex protons located closest to the $\mathrm{V}^{4+}$ center do not participate in nuclear spin diffusion to their surrounding protons, as they are located inside the spin diffusion barrier. This is clearly the case with complex 1 . As the $R_{\mathrm{V}-\mathrm{H}}$ increases, the tendency of the complex protons to transfer the polarization to the solvent protons via spin diffusion slowly increases. Thus, the $\mathrm{T}_{\mathrm{DNP}}$ is prohibitively long in $\mathbf{1}$, long in $\mathbf{2}$, short in 3, and even shorter in 4, implying that the spin diffusion rate increases and the polarization buildup time shortens from complexes $\mathbf{2 , 3}$ to $\mathbf{4}$. To rule out the effect of freezing procedure on $T_{D N P}$, a standardized freeze-quenching procedure was followed for all the measurements, and the reproducibility of these results were tested using the DNP frequency profiles, as discussed in ESI section 6. Furthermore, this behavior could be modeled numerically using the SpinEvolution software ${ }^{35}$ that is based on quantum mechanical calculations. Our model, based on a threespin system (one electron and two protons) shows that increasing the hyperfine interaction strength between the paramagnetic center and closest nuclear spins slows down the buildup rate of the remote nuclear spins (ESI Figure S9). The same trend observed in the $T_{1 n}$ values (see Table S3 of ESI) reinforces the conclusion that the rate of spin diffusion between the complex and solvent protons increases from complex
1 to 4. Note that the complex protons from compounds $\mathbf{2}$ and $\mathbf{3}$ are invisible to NMR due their short $T_{2 n}$ values; however, they still participate in the spin diffusion process as indicated by the trend of $T_{1 n}$ and $T_{D N P}$ values.

In complex 1, the EPR signal intensity is weak compared to the other three complexes (see Figure S1). We propose that the loss in signal intensity arises from its aggregation in the DMF solvent. Within these aggregates, a significant amount of $\mathrm{V}^{4+}$ ions are EPRsilent due to their extremely short $T_{m}$. While this may affect the DNP process, we also observed that $\mu \mathrm{w}$ off ${ }^{1} \mathrm{H}$ NMR signal in complex $\mathbf{1}$ is smaller compare to the other complexes (see Figure 3). We hypothesize that the protons of the counterions charge balancing the aggregated complex $\mathbf{1}$ are also becoming NMR-silent, once in close proximity to the paramagnetic sites in the clustered moieties. In other words, the clustering causes reduction in the effective $\mathrm{V}^{4+}$ ion concentration and reduces the effective ${ }^{1} \mathrm{H}$ concentration in the sample, as the ${ }^{1} \mathrm{H}$ 's in the clustered moieties do not participate in the spin-diffusion and NMR signal. Therefore, aggregated complex 1 does not show up in NMR, EPR and DNP measurements, effectively reducing the concentration of the complex. Since these effects only serve to reduce the concentration of the non-aggregated species, the ratio of proton spins to the paramagnetic centers has not significantly changed. Thus our observation of no DNP enhancement in complex $\mathbf{1}$ is consistent with the lack of spin-diffusion from complex proton to the solvent protons. These observations, taken together, allow us to conclude that the spin-diffusion barrier lies between $4.0 \AA$ and $6.6 \AA$ from the paramagnetic center. Additionally, through the differing rates of spin polarization from 2-4, we observe the "rate-limiting" influence of the spin diffusion barrier extending up to a distance of $12.6 \AA$ from the $\mathrm{V}^{4+}$ center.

The size of this spin diffusion barrier can be further verified using the phase memory relaxation time $\left(T_{m}\right)$ of the $\mathrm{V}^{4+}$ ion of all four complexes at comparable concentrations. The $T_{m}$ values acquired at $8.63 \mathrm{~T}$ and at $5 \mathrm{~K}$ are shown in Figure 5, and plotted together with the $T_{m}$ values published previously ${ }^{1}$ at $0.35 \mathrm{~T}$ and at 40 $\mathrm{K}$. In both the cases, the $T_{m}$ is longest for complex 1 as 
the nearest nuclear spins are well inside the spin diffusion barrier, and hence do not participate in facilitating the electron spin decoherence. ${ }^{1}$

(a)

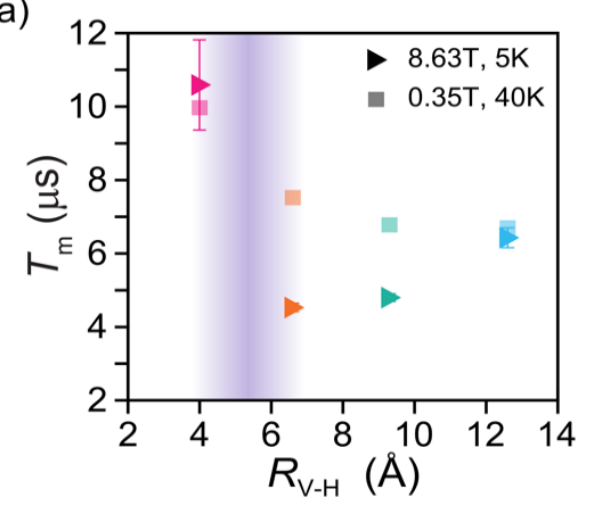

(b)

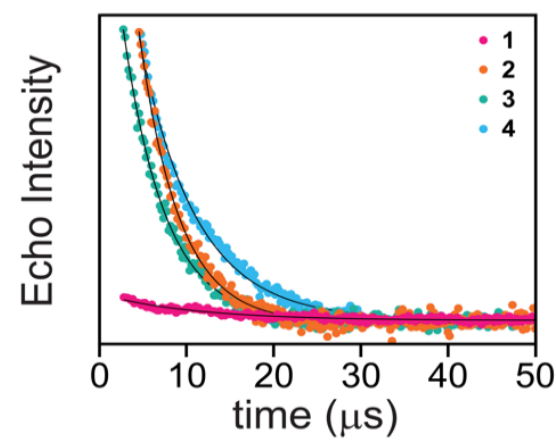

Figure 5 (a) Phase memory relaxation time constants $\left(T_{m}\right)$ for 1-4 in magenta, orange, green and cyan colors respectively, measured at $8.63 \mathrm{~T}$ field and $5 \mathrm{~K}$ temperature (triangles), $0.35 \mathrm{~T}$ and $40 \mathrm{~K}$ (squares) in saturated solutions. (b) The experimental data (plot markers) and the fitted exponential decay curves (cyan lines) to determine $T_{m}$ for complexes 1-4 at $8.63 \mathrm{~T}$ field and $5 \mathrm{~K}$ temperature.

At $0.35 \mathrm{~T}$ the $T_{m}$ values decreased significantly from 1 to 2 from $9.97 \mu$ s to $7.52 \mu$ s and then plateaued to $6.67 \mu$ s for 4. At $8.63 \mathrm{~T}$, we observed an even sharper decay in $T_{m}$ from $10.59 \mu$ s for complex 1 to $4.53 \mu$ s for complex 2, but after that the $T_{m}$ increased again for complex 3 with $4.8 \mu$ s and complex 4 with $6.43 \mu$ s. To confirm that the short $T_{m}$ is not a result of high concentration of $\mathbf{2}$, its $T_{m}$ was re-measured of sample 2 at a concentration of $13 \mathrm{mM}$ at $6.9 \mathrm{~T}$ and $4 \mathrm{~K}$, and found to be $3.0 \pm 0.15 \mu \mathrm{s}$, as shown in ESI Figure S11. The similarly short $T_{m}$ at both concentrations verifies that the observed decay in $T_{m}$ from $\mathbf{1}$ to $\mathbf{2}$ is due to the intrinsic $T_{m}$ of 2 , not $V^{4+}$ concentration. The trend in $T_{m}$ suggests that the nuclear spins located at distances in the range of $6 \AA$ to $10 \AA$ from the $V^{4+}$ center are the greatest contributors to the decoherence of the $\mathrm{V}^{4+}$ electron spins. Nuclear spins locate further are too weakly coupled to $\mathrm{V}^{4+}$, and nuclear spins located closer than this fall inside the spin-diffusion barrier, and do not participate in decoherence. Thus, the size of the spin diffusion barrier and the spin diffusion rates indicated by the DNP buildup curves and enhancements are in agreement with these results.

\section{Conclusions}

This study demonstrates that significant NMR signal enhancements at high magnetic fields (6.9 T) can be induced by DNP using wide line transition metals ions $\left(\mathrm{V}^{4+}\right)$ with $g$-values differing significantly from 2 as the polarizing centers for DNP. We confirmed the spin diffusion barrier for the ${ }^{1} \mathrm{H}$ nuclear spins to lie at a distance of $4.0 \AA$ to $6.6 \AA$ from the $V^{4+}$ center, but that the effect of the spin diffusion barrier is felt by the nearest $1 \mathrm{H}$ spin located up to $12.6 \AA$ distance away from $\mathrm{V}^{4+}$ center. We identified the preferred polarization pathway in DNP under our experimental conditions to be via nuclear spin diffusion from the complex protons, located at distances $6.4 \AA$ to $12.6 \AA$ from the paramagnetic metal center to the surrounding solvent protons. The experimental results show that the DNP buildup rates is determined by the rate of nuclear spin-diffusion from the nuclear spin nearest to the paramagnetic center to the bulk nuclear spins. Additionally, we demonstrate that direct DNP as far as $12.6 \AA$ from a paramagnetic transition metal is viable. This supports the existing hypothesis that a depleted nuclear spin density inside the spin diffusion barrier of a paramagnetic center can facilitate efficient DNP buildup pathway, extending the range of direct transfer.

Notably, the foregoing experiments are the first demonstration of DNP using $\mathrm{V}^{4+}$ metal sites as polarizing agents. Expanding our catalog of polarizing agents offers the potential to enable endogenous polarization within molecules and materials of interest. Specifically, vanadium as the catalytically active species in both heterogeneous catalysis systems, such as vanadium oxides, and in metalloenzymes, including vanadium 
haloperoxidase..$^{36-37}$ This proof-of-concept study demonstrates a pathway to harness paramagnetic vanadium centers within catalytic systems to selectively enhance and illuminate the NMR signal sensitivity within the first and second coordination spheres of chemically-active vanadium sites. By using endogenous polarization, DNP-enhanced hyperfine NMR spectroscopy has the potential to couple high sensitivity with local structural information.

\section{Conflicts of interest}

There are no conflicts to declare.

\section{Acknowledgements}

We thank Dr. Asif Equbal for performing the quantum mechanical simulations using SpinEvolution that are shown in ESI Figure S9. We are thankful to Dr. Ilia Kaminker for the fruitful discussions on the experimental data and manuscript. We thank M. J. Graham and K. A. Collins for experimental assistance. D.E.F and C.-J. Y. acknowledge NSF CHE-1455017 for funding the synthetic component of this research. S. K. J, T.T. and S.H. acknowledge NSF CHE-1505038 for partial support, S.H. acknowledges the NIH R21EB022731 for the AWG-DNP setup, and B.W. and S.H. acknowledge NSF MCB-1617025 for EPR studies at 240 $\mathrm{GHz}$. Part of this work was performed at the ITST Terahertz Facilities at UCSB, which have been upgraded under NSF Award No. DMR-1126894

\section{Notes and references}

(1) Graham, M. J.; Yu, C.-J.; Krzyaniak, M. D.; Wasielewski, M. R.; Freedman, D. E., J. Am. Chem. Soc. 2017, 139, 3196-3201.

(2) Lilly Thankamony, A. S.; Wittmann, J. J.; Kaushik, M.; Corzilius, B., Prog. Nucl. Magn. Reson. Spectrosc. 2017, 102-103, 120-195.

(3) Corzilius, B.; Smith, A. A.; Barnes, A. B.; Luchinat, C.; Bertini, I.; Griffin, R. G., J. Am. Chem. Soc. 2011, 133, 5648-5651.

(4) Kaushik, M.; Bahrenberg, T.; Can, T. V.; Caporini, M. A.; Silvers, R.; Heiliger, J.; Smith, A. A.; Schwalbe, H.; Griffin, R. G.; Corzilius, B., Phys. Chem. Chem. Phys. 2016, 18, 27205-27218.
(5) Corzilius, B.; Michaelis, V. K.; Penzel, S. A.; Ravera, E.; Smith, A. A.; Luchinat, C.; Griffin, R. G., J. Am. Chem. Soc. 2014, 136, 11716-11727.

(6) Corzilius, B., Paramagnetic Metal Ions for Dynamic Nuclear Polarization. In eMagRes, Harris, R. K.; Wasylishen, R. L., Eds. Wiley \& Sons, Ltd: 2018; Vol. 7. (7) Wenk, P.; Kaushik, M.; Richter, D.; Vogel, M.; Suess, B.; Corzilius, B., J. Biomol. NMR 2015, 63, 97-109.

(8) Wolf, T.; Kumar, S.; Singh, H.; Chakrabarty, T.; Aussenac, F.; Frenkel, A. I.; Major, D. T.; Leskes, M., J. Am. Chem. Soc. 2018.

(9) Bloembergen, N., Physica 1949, 15, 386-426.

(10) Blumberg, W. E., Phys. Rev. 1960, 119, 79-84.

(11) Takahashi, S.; Hanson, R.; van Tol, J.; Sherwin, M. S.; Awschalom, D. D., Phys. Rev. Lett. 2008, 101, 047601.

(12) McConnell, H. M.; Chesnut, D. B., J. Chem. Phys. 1958, 28, 107-117.

(13) Kurland, R. J.; McGarvey, B. R., J. Magn. Reson. (1969) 1970, 2, 286-301.

(14) Pennanen, T. O.; Vaara, J., Phys. Rev. Lett. 2008, 100, 133002.

(15) Clore, G. M.; Iwahara, J., Chem. Rev. 2009, 109, 4108-4139.

(16) Corzilius, B.; Andreas, L. B.; Smith, A. A.; Ni, Q. Z.; Griffin, R. G., J. Magn. Reson. 2014, 240, 113-123.

(17) Rogawski, R.; Sergeyev, I. V.; Zhang, Y.; Tran, T. H.; Li, Y.; Tong, L.; McDermott, A. E., J. Phys. Chem. B 2017, 121, 10770-10781.

(18) Rogawski, R.; Sergeyev, I. V.; Li, Y.; Ottaviani, M. F.; Cornish, V.; McDermott, A. E., J. Phys. Chem. B 2017, 121, 1169-1175.

(19) Marin-Montesinos, I.; Goyard, D.; Gillon, E.; Renaudet, O.; Imberty, A.; Hediger, S.; De Paëpe, G., Chemical Science 2019.

(20) Khutsishvili, G., Soviet Physics JETP 1962, 15.

(21) Smith, A. A.; Corzilius, B.; Barnes, A. B.; Maly, T.; Griffin, R. G., J. Chem. Phys. 2012, 136, 015101.

(22) Barnes, A. B.; Corzilius, B.; Mak-Jurkauskas, M. L.; Andreas, L. B.; Bajaj, V. S.; Matsuki, Y.; Belenky, M. L.; Lugtenburg, J.; Sirigiri, J. R.; Temkin, R. J.; Herzfeld, J.; Griffin, R. G., Phys. Chem. Chem. Phys. 2010, 12, 58615867.

(23) Leavesley, A.; Jain, S.; Kamniker, I.; Zhang, H.; Rajca, S.; Rajca, A.; Han, S., Phys. Chem. Chem. Phys. 2018, 20, 27646-27657.

(24) Hu, K.-N.; Debelouchina, G. T.; Smith, A. A.; Griffin, R. G., J. Chem. Phys. 2011, 134, 125105.

(25) Tan, K. O.; Mardini, M.; Yang, C.; ArdenkjærLarsen, J. H.; Griffin, R. G., Science Advances 2019, 5, eaax2743. 
(26) Siaw, T. A.; Leavesley, A.; Lund, A.; Kaminker, I.; Han, S., J. Magn. Reson. 2016, 264, 131-153.

(27) Leavesley, A.; Kaminker, I.; Han, S., Versatile DNP Hardware with Integrated EPR. In Dynamic Nuclear Polarization Handbook, Michaelis, V.; Griffin, R. G.; Vega, S.; Corzilius, B., Eds. Wiley \& Sons, Ltd: 2018.

(28) Kaminker, I.; Barnes, R.; Han, S., J. Magn. Reson. 2017, 279, 81-90.

(29) Stoll, S.; Schweiger, A., J. Magn. Reson. 2006, 178, 42-55.

(30) Wenckebach, W. T., Appl. Magn. Reson. 2008, 34, 227.

(31) Kaminker, I.; Han, S., J. Phys. Chem. Lett. 2018, 9, 3110-3115.

(32) We hypothesize that the solubility of complex 2 in DMF is particularly high due to the optimal balance between the non-polar nature of the organic ligands and the overall charge of the complex. With the longer linkers in complex 3 and 4, the nonpolar nature of those ligands are dominant and inhibit solubility in DMF. With complex 1 , the ligand is more ionic in nature, and combined with the 2- charge of the complex may render the complex highly ionic, hence reducing its solubility in DMF. Taken together, we propose that the nonpolar nature of the ligands on complex 2 balance the ionic nature of the complex, thereby enhancing its solubility in DMF.

(33) Morse, D. B.; Hendrickson, D. N.; Rauchfuss, T. B.; Wilson, S. R., Organometallics 1988, 7, 496-502.

(34) Tse, D.; Hartmann, S. R., Phys. Rev. Lett. 1968, 21, 511-514.

(35) Veshtort, M.; Griffin, R. G., J. Magn. Reson. 2006, 178, 248-282.

(36) Weckhuysen, B. M.; Keller, D. E., Catal. Today 2003, 78, 25-46.

(37) Messerschmidt, A.; Wever, R., Proc. Nat. Aca. Sci. 1996, 93, 392. 